\title{
Airflow relieves chronic breathlessness in people with advanced disease: an exploratory systematic review and meta-analyses.
}

Flavia Swan, Wolfson Palliative Care Research Centre, Institute for Clinical and Applied Health

Research, Allam Medical Building, Hull York Medical School (HYMS), University of Hull,

Cottingham Rd, Hull, HU6 7RX

Alison Newey, Community Palliative Care, University Hospital of South Manchester NHS

Foundation Trust, Withington Community Hospital, Nell Lane, Manchester, M20 2LR

Martin Bland, Department of Health Sciences, Seebohm Rowntree Building, University of York, Heslington, York YO10 5DD

Victoria Allgar, Department of Health sciences, University of York, Heslington, York YO10 5DD

Sara Booth, Associate Lecturer, Department of Oncology, University of Cambridge, Cambridge CB2 OQQ

Claudia Bausewein, Department of Palliative Medicine, Munich University Hospital, Marchioninistr. 15, 81377 München, Germany

Janelle Yorke, School of Health Sciences, Division Nursing, Midwifery and Social Work, University of Manchester. Room 5.320, Jean McFarlane University, University Place, Oxford Road, Manchester M13 9PL and Christie Patient Centred Research group (CPCR) The Christie NHS Foundation Trust, Manchester.

Miriam Johnson, Wolfson Palliative Care Research Centre, Institute for Clinical and Applied Health Research, Allam Medical Building, Hull York Medical School (HYMS), University of Hull, Cottingham Rd, Hull, HU6 7RX 


\section{Abstract}

Background: Chronic breathlessness is a neglected symptom of advanced diseases. Aim: To examine the effect of airflow for chronic breathlessness relief.

Design: Exploratory systematic review and meta-analysis.

Data sources: Medline, CINAHL, AMED and Cochrane databases were searched (1985 2018) for observational studies or randomised controlled trials of airflow as intervention or comparator. Selection against pre-defined inclusion criteria, quality-appraisal and data extraction were conducted by two independent reviewers with access to a third for unresolved differences. "Before and after” breathlessness measures from airflow arms were analysed. Meta-analysis was carried out where possible.

Results: 16/78 studies ( $\mathrm{n}=929)$ were included; 11 randomised controlled trials of oxygen vs medical air, four randomised controlled trials and one fan cohort study. Three meta-analyses were possible: i) Fan at rest in three studies (n=111) offered significant benefit for breathlessness intensity (0-100mm Visual Analogue Scale and 0- 10 Numerical Rating Scale), mean difference -11.17 (95\% confidence intervals -16.60 to -5.74 ), $p=0.06 I^{2} 64 \%$. ii) Medical air via nasal cannulae at rest in two studies $(n=89)$ improved breathlessness intensity (visual analogue scale), mean difference $-12.0 \mathrm{~mm}$, 95\% confidence intervals -7.4 to -16.6 , $\mathrm{P}<0.0001 \mathrm{I}^{2}=0 \%$. iii) Medical airflow during a constant load exercise test before and after rehabilitation ( $\mathrm{n}=29)$ in two studies improved breathlessness intensity (mBorg, 0-10) mean difference $-2.9,95 \%$ confidence intervals -3.2 to $-2.7, \mathrm{p}<0.0001 \mathrm{I}^{2}=0 \%$.

Conclusion: Airflow appears to offer meaningful relief of chronic breathlessness and should be considered as an adjunct treatment in the management of breathlessness.

\section{Keywords:}

- dyspnea,

- self-management,

- review,

- airflow (relevant term as the intervention subject heading) 


\section{What is already known?}

- Randomised controlled trials and cohort data have demonstrated that airflow delivered from the fan at rest offers significant relief of breathlessness.

- Systematic review and randomised controlled trials of oxygen $v s$ medical air have failed to demonstrate additional benefit from oxygen therapy and suggest that medical air delivery, airflow, is likely to be an active intervention.

- All current evidence available for the effect of airflow for chronic breathlessness relief has not been explored using systematic review methods.

\section{What this paper adds}

This exploratory systematic review and meta-analyses provide promising data to suggest that:

- airflow from the fan at rest improves breathlessness in people with breathlessness due to a variety of causes

- airflow delivered as cylinder medical air at rest improves breathlessness in advanced cancer

- airflow delivered as cylinder medical air during a constant load exercise test in people with chronic obstructive pulmonary disease and who have completed pulmonary rehabilitation

\section{Implications for practice and theory}

- Clinicians should consider the fan as an adjunct to treatment for breathlessness at rest in patients who do not require oxygen-enriched air.

- Airflow may benefit exertion-induced breathlessness, but further work is required to investigate the role of the fan with everyday general activity and in relation to exercise.

- Recovery time from exertion-induced breathlessness, self-efficacy and daily activity are key outcomes to explore in future studies of airflow. 


\section{Introduction}

Breathlessness is a common, often poorly managed symptom in people with advanced diseases. It is associated with reduced quality of life (1), decreased activities of daily living (2), unplanned emergency hospital attendance and admission. (3-5) Breathlessness inflicts devastating and disabling physical, psychological and social burden on normal daily life for the patient, carers and close family members (6-8). Chronic breathlessness, that is, breathlessness that persists despite optimal treatment for the underlying pathophysiology and causing such disability (9), all too often is left for patients to manage themselves despite a developing evidence base for interventions targeted at the breathlessness itself.

Growing evidence supports complex non-pharmacological interventions to reduce the impact of the symptom and improve quality of life. (10-12) Components target peripheral and central afferent sources of breathlessness sensation, such as facial airflow delivered by the batteryoperated hand-held fan (fan). (13-17) Cooling of the facial skin innervated by the $2^{\text {nd }}$ and $3^{\text {rd }}$ branches of the trigeminal nerve, nasal mucosae or the upper airway flow receptors could modulate the central perception of breathlessness leading to decreased neural respiratory drive, thereby reducing the sensation of breathlessness. (18-22) A recent multi-methods secondary analysis of qualitative interview data from three studies found that 80/111 (72\%) participants experienced benefit when the fan was used in conjunction with other components of a complex intervention. (23) Airflow delivered from the fan may offer a valuable contribution to the self-management of chronic breathlessness $(13,15,23)$, and has been identified as a potentially useful strategy in a variety of situations, e.g. breathlessness crisis (24), a component of pulmonary rehabilitation to assist recovery from exercise, or with general everyday activities. (15)

Systematic reviews of oxygen in a variety of non-hypoxic patient groups (cancer, chronic heart failure, kyphoscoliosis, chronic obstructive pulmonary disease and interstitial lung disease) have not demonstrated additional benefit from oxygen therapy over medical air delivery. (25-30) An updated Cochrane review of chronic obstructive pulmonary disease found low quality evidence for modest relief of breathlessness. (31) The results from a large, adequately powered trial that randomised 239 participants (chronic obstructive pulmonary disease $63 \%$, cancer $16 \%$ ) to receive at least 15 hours a day of oxygen or medical air delivered via home concentrator for seven days reaffirms earlier suggestion that medical air 
used in the placebo arm may not be an inert comparator as previously thought and points to the likelihood of an active intervention. $(29,32)$ Therefore the placebo arm of oxygen studies may provide useful preliminary data regarding the role of airflow for the relief of chronic breathlessness. This systematic review aims to identify and evaluate data from studies of airflow, both from studies of the hand-held fan and the comparator arm data for breathlessness intensity from oxygen studies, analysed as "before and after" airflow exposure cohort data.

\section{Aim}

To examine the current evidence for the effectiveness of airflow for the relief of chronic breathlessness.

\section{Methods}

The systematic review methods employed an exploratory approach in that only the airflow arm of studies were used and the data analysed as cohort "before and after" treatment.

\section{Study design}

The search methods employed are adapted from the Cochrane Handbook of Systematic Reviews (33) and reported in accordance with the Preferred Reporting Items for Systematic Reviews and Meta-Analyses. (34) A review protocol is not pre-registered but available from the University of Hull Library (Flavia Swan PhD Thesis).

\section{Inclusion and exclusion criteria}

\section{Types of studies}

Randomised controlled trials, controlled clinical trials (quasi-randomised experimental trials with or without blinding) and observational cohort studies were included. 


\section{Types of participants}

Adults with chronic breathlessness from any advanced disease aetiology as shown below:

- Malignancy: advanced primary and metastatic cancer patients, who have undergone disease treatments like chemotherapy, radiotherapy or surgical interventions.

- Chronic Obstructive Pulmonary Disease with forced expiratory volume in 1 second of less than $50 \%$ predicted value

- Interstitial lung disease or pulmonary fibrosis where breathlessness is present

- Chronic heart failure: New York Heart Association stage III-IV

- Motor Neurone disease and other neurological disease where breathlessness is present or forced vital capacity less than $80 \%$ predicted value

- Kyphoscoliosis: a moderate - severe sideways and forwards curvature of the spine Cobb Angle $>50^{\circ}$ and forced expiratory volume of less than $50 \%$ predicted value.

Studies were included if at least $50 \%$ of the study population were classified as advanced, palliative or in the later stages of disease as defined above. These criteria were adapted from the Cochrane review of non-pharmacological interventions for breathlessness. (35)

Studies of participants with mild hypo or normoxaemia, who do not fore-fill the criteria for long term oxygen therapy (36) were included. Studies of hypoxic participants or patients with any condition not assessed as progressive, refractory to treatment and advanced such as asthma were excluded.

\section{Types of exposure}

Airflow: i) delivered from either a fan (hand-held or table) or non-oxygen enriched compressed air, or from a non-invasive ventilatory method (nasal cannula, mask or mouthpiece), but not nasal intermittent positive pressure ventilation and ii) directed at the cheek of the face, nasal mucosae or mouth.

Administration: as i) a single dose during ambulation, or at rest taken as needed (pro re nata),(37) ii) placebo short-burst oxygen therapy intermittent use before exercise or after 
exercise for recovery (36) or iii) continuously over $15 \mathrm{hr}$ a day as placebo long-term oxygen therapy studies or during the night as placebo nocturnal oxygen therapy.(38)

Studies where airflow was directly administered to the trachea, or at sub-zero temperatures were excluded.

\section{Types of outcome measure}

\section{Unidimensional breathlessness outcomes}

ATS domains of dyspnea measurement (20) including breathlessness severity or intensity rated on uni-dimensional scales as shown below:

- Modified Borg Score, a categorical scale with ratio properties

- Visual Analogue Scale, 0 - 100mm anchored $0=$ no shortness of breath and $100 \mathrm{~mm}=$ shortness of breath as bad as can be

- Numerical Rating Scales, 0-10 numbered scale anchored $0=$ Not breathless at all and 10 the worst imaginable breathlessness

- Likert scales with verbal responses such as "a bit better”, “much better” or "no difference” or any other validated uni-dimensional scale for measuring breathlessness.

Studies were only included if they reported the breathlessness outcome at baseline and posttreatment measured as either primary or secondary outcomes. If severity or intensity was measured as part of a multi-dimensional or composite scale, e.g. the Chronic Respiratory Questionnaire, that unidimensional measure of breathlessness was not extracted and analysed separately. Breathlessness related function/quality of life measures were not used as primary breathlessness outcomes in the absence of unidimensional scales. 


\section{Other Outcomes}

Other outcomes as shown below measured as either primary or secondary outcomes.

- Participant preference and satisfaction with the treatment

- Participant withdrawal and drop-out from the studies

- Adverse effects recorded

\section{Data sources and searches}

Medline, CINAHL, AMED and Cochrane databases were searched (1985 - 2015; updated January 2018) for observational or randomised controlled trials of airflow as intervention vs control or as comparator vs oxygen. Reference lists were scanned. A full search strategy can be seen in Online Supplementary Table 1.

\section{Study selection}

Titles, abstracts (and, where unclear, full papers) were screened against the eligibility criteria by two independent reviewers FS and $\mathrm{AN}$, with recourse to $\mathrm{MJ}$ as a third reviewer in case of disagreement.

\section{Data extraction and synthesis}

Baseline and post-intervention measures of breathlessness intensity were extracted from the fan studies and from the comparator arm of oxygen studies. Data were analysed as "before and after” airflow exposure cohort observational data.

\section{Risk of bias}

FS and AN judged the reporting quality and internal validity for each of the included studies. The cohort study was evaluated according to the Cochrane guidelines for assessing bias in a non-randomised study. (39) As there is no tool that is applicable directly to the data extracted from the randomised controlled trials control arms, we assessed instead the quality of the parent randomised controlled trials as a proxy marker for quality data. The randomised 
controlled trials were assessed with the Cochrane Risk of bias tool. (33) See online Supplementary Table 2.

\section{Statistical Analysis}

Results from the meta-analyses were reported for the primary outcome, breathlessness intensity or severity where heterogeneity allowed, or where not possible these were described narratively. Numerical rating scales and visual analogue scales were combined by equating one point on a numerical rating scales scale to $10 \mathrm{~mm}$ on a visual analogue scales. $(40,41)$

Data calculations for mean difference and standard deviation used STATA Version 12.1, Stata Corp LLC

Texas 77845-4512, USA. Breathlessness measurements were analysed as continuous outcomes. Data from the placebo arm of cross-over randomised controlled trials were treated as single arm before-after studies. For studies that recorded median values, the mean were calculated from the extracted study data. (42) The $I^{2}$ statistic was used to assess heterogeneity. (43) Where the result indicated significant heterogeneity a random effects model was chosen, otherwise a fixed effects model was applied. All analyses were undertaken on Review Manager 5.5. A sensitivity analysis was attempted for any study identified as including a sub-group not fitting the review criteria of mild hypo or normoxaemia to assess for any significant difference in the breathlessness outcome between the hypoxic and non-hypoxic participants.

\section{Results}

A total of 403 records were identified for screening. After removal of duplicates, 78 records were reviewed. 14 abstracts were rejected for not meeting inclusion criteria; the remaining 64 full text articles were assessed for eligibility. Of these, 16 studies met the review inclusion criteria and the other 48 studies were excluded (see Figure 1; flow chart (34) and Online Supplementary Table 3, eAppendix).

Overall studies represented 929 participants (age median 61.5, range 33 to 90 years; 47\% men) 


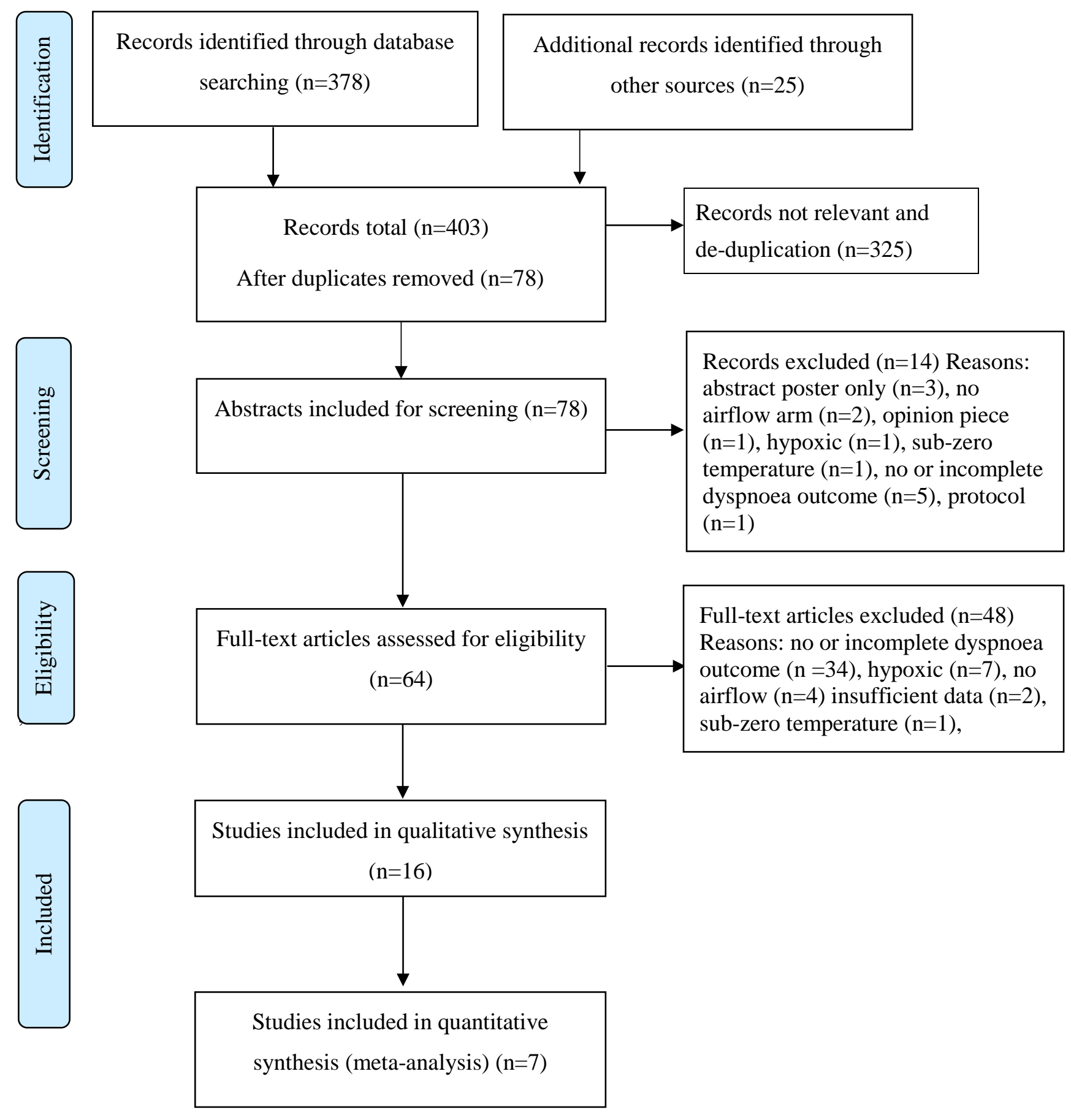

Figure 1 Flow-diagram of study selection and retrieval (34)

Airflow was delivered by fan $(13,14,16,17,44)$ or as medical air. $(29,32,45-53)$ See Table 1 for study characteristics 


\section{Description of fan studies}

Design: five studies ( $\mathrm{n}=230$ ) used the fan. Two feasibility randomised controlled trials; (n =49), (13) and ( $n=30),(44)$, a feasibility cohort study ( $n=31),(14)$, a feasibility longitudinal RCT ( $n=70),(16)$ and a phase III cross-over randomised controlled trial ( $n=50),(17)$.

Patient characteristics: Four studies recruited a mixed population of people with breathlessness due to a variety of advanced conditions including chronic obstructive pulmonary disease $(n=101)$, cancer $(n=55)$, heart failure $(n=23)$ and other causes $(n=21),(13$, $14,16,17)$, and one study recruited advanced cancer only $(n=30)$. (44)

Intervention and comparator characteristics: three studies used the fan to face at rest (14, $17,44)$, two with comparator groups; fan to leg (17), or no fan use and carer support (44), and the other was a cohort design. (14) One study assessed acceptability of the fan when used with general activity over 6 months compared with an acupressure wristband (16), and the remaining study assessed the fan when used with exercise advice over 4 weeks. (13)

Breathlessness Outcome: Three studies focused on the sensory-perceptual domain of dyspnea measurement and used breathlessness intensity as the primary outcome (17) or main outcome $(14,44)$. These studies selected the visual analogue scale (17), the numerical rating scale (44), or both visual analogue scale and numerical rating scale (14). The other two studies assessed symptom impact as well as the sensory-perceptual domain. These studies selected the numerical rating scale breathlessness intensity (13) and the Modified Borg Scale of breathlessness severity. (16)

Other outcomes: All of the fan studies reported participant withdrawals (13, 14, 16, 17, 44). These ranged from 0 to 6 participants. $(13,14,44)$ One study reported that there were no adverse events (13) and the other fan studies did not include any adverse event details. (14, $16,17,44)$ Airflow preferences were described in four fan studies $(13,16,17,44)$ and not in one study. (14) In addition, one study quantified the experience of fan use at 2 months. (16)

\section{Description of medical air studies}


Design: eleven randomised controlled trials ( $\mathrm{n}=699)$ used oxygen, helium hyperoxia or both gases for the intervention compared with medical air. (29, 32, 45-53) Study size ranged from 16 to 239 participants. $(29,48)$ Four were cross-over $(32,48,49,51)$ and seven used a parallel group design. (29, 45-47, 50, 52, 53) Nine studies were double blind (29, 45-52), and two were single blind. $(32,53)$

Patient characteristics: the eleven studies represent; chronic obstructive pulmonary disease $n=537$, cancer $n=109$, other lung diseases $n=21$, cardiac disease $n=14$ and other causes $n=18$. Inclusion criteria required moderate to severe chronic obstructive pulmonary disease (45-50, 52,53 ), advanced cancer (51), or were a mixed population with no specific stipulation of severity. $(29,32)$

Intervention Characteristics: the source of airflow was an oxygen cylinder (32, 45-50), a sham concentrator (29), and a Douglas bag. (52) Two studies did not state the airflow source. $(51,53)$ Medical air or compressed air was delivered through nasal cannulae (29, 32, 45, 47, 49-51, 53), face-mask and nasal cannula (48), a non-rebreathing face-mask (46) and through a mouthpiece. (52) The flow rates varied widely in the studies; 2l/minute (29, 45), 3l/minute (47), $41 /$ minute (32, 49, 51), 5l/minute (53), 6l/minute (50) and 81/minute via nasal cannula or 15l/minute with face mask. (48) Two studies did not report flow rate details. $(46,52)$ The timing of airflow delivery was; 15 minutes at rest (32, 51), with daily activity over 3 (50), or 6 months (45), 15 hours a day over one week (29), or in conjunction with exertion-induced breathlessness during pulmonary rehabilitation, $(46,52,53)$ or a walking test. $(47-49)$ The pulmonary rehabilitation programme parameters for airflow delivery were with treadmill exercise 3 times a week for 30 minutes over two months (53), a cycle ergometer used 3 times a week for 30 minutes over 6 weeks (46), or 3 times a week for 20 minutes over two months. (52) The 6minute walk test parameters for airflow delivery were; i) three same day 6minute walk test s with 45 minutes washout, using room air for the basal walk and compressed air for the subsequent walks (47), ii) five 6minute walk test performed over three visits, (timing not stated) using room air for the practice walk on visit one and cylinder air for the two 66minute walk test s with 60 minute washout on visits two and three (48), and iii) three same day 6minute walk test s using cylinder air with 20 minutes washout between tests at baseline, 6 and 12 weeks as well as short burst use at home with daily activity during the study period. 
Breathlessness outcome: two studies focused on the sensory-perceptual domain of dyspnea measurement and recorded breathlessness intensity as a primary outcome with the visual analogue scale and Borg scale (32) or the visual analogue scale only. (51) All of the other studies focused on symptom impact as well as the sensory-perceptual domain. (29, 45-50, 52, 53) Of these, three studies measured breathlessness intensity as a primary outcome with the numerical rating scale (29) or the Chronic Respiratory Questionnaire dyspnea domain. (45, 50) The remaining six studies identified the modified Borg scale as one of the main outcomes $(47-49,52,53)$ or a secondary measure. (46) One study in addition selected the Chronic Respiratory Questionnaire. (49)

Other outcomes: participant withdrawals were reported in all of the studies (29, 32, 45-51, 53), apart from one. (52) Five studies reported no withdrawals (32, 46-48, 51) and in the other five studies withdrawals ranged from 2 to 21 participants. $(45,53)$ adverse events were poorly reported with only two studies including details; "few" or "no adverse events". (29, 46) All of the other studies omitted reporting adverse events. (32, 45, 47-53) Airflow preferences were only reported in one study. (51) The remaining studies did not report airflow preferences $(29,32,45-48,50,52,53)$, although one study did quantify side-effects (29) and a second study examined preference for cylinder delivery of airflow. (50) 
Table 1 Characteristics of included studies (fan)

\begin{tabular}{|c|c|c|c|c|c|c|c|c|c|c|}
\hline $\begin{array}{l}\text { Study } \\
\text { author }\end{array}$ & Study Design & $\begin{array}{l}\text { Population } \\
\text { (mean; standard } \\
\text { deviation) }\end{array}$ & Intervention & Comparator & $\begin{array}{l}\text { Mode } \\
\text { of gas } \\
\text { delivery }\end{array}$ & $\begin{array}{l}\text { Dyspnea } \\
\text { Outcome } \\
\text { measure(s) }\end{array}$ & $\begin{array}{l}\text { Other Outcomes: } \\
\text { withdrawals, Adverse } \\
\text { Events (AE), airflow } \\
\text { preferences }\end{array}$ & $\begin{array}{l}\text { Timing of } \\
\text { measure } \\
\text { ment }\end{array}$ & $\begin{array}{l}\text { Results airflow arm only } \\
\text { (before and after } \\
\text { treatment) } \\
\text { (mean; standard deviation) }\end{array}$ & $\begin{array}{l}\text { Improvement } \\
\text { with airflow } \\
\text { Yes/No }\end{array}$ \\
\hline $\begin{array}{l}\text { Booth } \\
\text { (2016) [14] }\end{array}$ & $\begin{array}{l}\text { Feasibility } \\
\text { observational } \\
\text { cohort }\end{array}$ & $\begin{array}{l}\mathrm{n}=31 \text { Males: } 20 \\
\text { Age mean: } 74.8 ; 11.49 \\
\text { Mixed population, non- } \\
\text { malignant, } \\
\text { cardiorespiratory } \\
\text { disease: } 8 \text { (26\%) } \\
\text { Baseline dyspnoea } \\
\text { score: } \\
\text { Mean Visual analogue } \\
\text { scale } 48 \mathrm{~mm} ; 27.4\end{array}$ & $\begin{array}{l}\text { Hand-held } \\
\text { fan to face }\end{array}$ & $\begin{array}{l}\text { No } \\
\text { comparator } \\
\text { group }\end{array}$ & $\begin{array}{l}\text { Airflow } \\
\text { from } \\
\text { hand- } \\
\text { held } \\
\text { fan to } \\
\text { face for } \\
5 \\
\text { minutes }\end{array}$ & $\begin{array}{l}\text { Visual } \\
\text { analogue } \\
\text { scale } \\
(\mathrm{mm}), \\
\text { numerical } \\
\text { rating scale }\end{array}$ & $\begin{array}{l}\text { Withdrawals = } 6 \\
\text { AE and airflow } \\
\text { preferences not } \\
\text { reported }\end{array}$ & $\begin{array}{l}\text { After } 5 \\
\text { minutes } \\
\text { at rest }\end{array}$ & $\begin{array}{l}\text { Visual analogue scale = } \\
\text { Mean } 35 \mathrm{~mm} ; 25.7 \\
\text { after } 5 \mathrm{~min} \text { air } \\
\text { Mean change = } 12 \mathrm{~mm} \text {; } \\
21.2\end{array}$ & Yes \\
\hline $\begin{array}{l}\text { Bausewein } \\
\text { (2010) [16] }\end{array}$ & $\begin{array}{l}\text { Feasibility } \\
\text { longitudinal } \\
\text { phase II } \\
\text { randomised } \\
\text { controlled trial }\end{array}$ & $\begin{array}{l}\mathrm{n}=70 \text { Males: } 36 \\
\text { Age mean: } 65.6 y r s \mathrm{SD} \\
8.80 \text { chronic obstructive } \\
\text { pulmonary disease }=45 \text {, } \\
\text { cancer }=25 \\
\text { Baseline dyspnoea } \\
\text { score: } 3.7 ; 1.83\end{array}$ & $\begin{array}{l}\text { Hand-held } \\
\text { fan to face }\end{array}$ & Wristband & $\begin{array}{l}\text { Airflow } \\
\text { from } \\
\text { hand- } \\
\text { held } \\
\text { fan }\end{array}$ & $\begin{array}{l}\text { Modified } \\
\text { Borg score }\end{array}$ & $\begin{array}{l}\text { Withdrawals at } 2 \\
\text { months }=16 / 33(48 \%) \\
\text { AE not reported } \\
\text { Airflow preferences: } \\
\text { Positive }=13 / 38 \\
\text { Negative }=7 / 38\end{array}$ & $\begin{array}{l}\text { Monthly } \\
\text { over } 6 \\
\text { months }\end{array}$ & $\begin{array}{l}\text { Mean Borg score change } \\
\text { over } 2 \text { months }=0.6 ; 2.1, p \\
=0.90\end{array}$ & $\begin{array}{l}\text { No, phase II } \\
\text { not powered to } \\
\text { test }\end{array}$ \\
\hline $\begin{array}{l}\text { Galbraith } \\
\text { (2010) [17] }\end{array}$ & $\begin{array}{l}\text { Cross-over } \\
\text { randomised } \\
\text { controlled trial }\end{array}$ & $\begin{array}{l}\mathrm{n}=50 \text { Males: } 23 \\
\text { Age mean: } 71.3 \text {, range } \\
\text { 33-90yrs } \\
\text { Mixed population; chronic } \\
\text { obstructive pulmonary } \\
\text { disease }=26, \text { lung } \\
\text { cancer }=11 \text {, heart } \\
\text { disease = } 15 \\
\text { Baseline dyspnoea } \\
\text { score: }\end{array}$ & $\begin{array}{l}\text { Hand-held } \\
\text { fan to face }\end{array}$ & $\begin{array}{l}\text { Hand-held } \\
\text { fan to leg }\end{array}$ & $\begin{array}{l}\text { Airflow } \\
\text { from } \\
\text { hand- } \\
\text { held } \\
\text { fan to } \\
\text { face for } \\
5 \\
\text { minutes }\end{array}$ & $\begin{array}{l}\text { Visual } \\
\text { analogue } \\
\text { scale (mm) }\end{array}$ & $\begin{array}{l}\text { Withdrawals = } 1 \\
\text { AE not reported } \\
\text { Airflow preferences: } \\
\text { positive patient } \\
\text { comments, numbers } \\
\text { not reported }\end{array}$ & $\begin{array}{l}\text { After } 5 \\
\text { minutes } \\
\text { at rest } \\
\text { and after } \\
10 \\
\text { minute } \\
\text { washout }\end{array}$ & $\begin{array}{l}\text { Visual analogue scale = - } \\
7.0 \mathrm{~mm} \text { Median change } \\
\text { after } 5 \text { minutes Fan/face } \\
\text { 1st group (interquartile } \\
\text { range } 1.5 \text { - 14.5) } \\
\text { Visual analogue scale = } \\
-10.0 \mathrm{~mm} \text { Median change } \\
\text { incl } 10 \text { minute washout } \\
\text { Fan/face 1st group }\end{array}$ & Yes \\
\hline
\end{tabular}




\begin{tabular}{|c|c|c|c|c|c|c|c|c|c|c|}
\hline & & $\begin{array}{l}\text { Visual analogue scale } \\
\text { Fan/face } 1 \mathrm{st} \text { group = } \\
31 \mathrm{~mm} ; 12-61 \mathrm{~mm}\end{array}$ & & & & & & & $\begin{array}{l}\text { (interquartile range } 3.5- \\
\text { 17), } P=0.003\end{array}$ & \\
\hline $\begin{array}{l}\text { Johnson } \\
\text { (2016) [13] }\end{array}$ & $\begin{array}{l}\text { Feasibility } \\
\text { phase II } \\
\text { randomised } \\
\text { controlled trial }\end{array}$ & $\begin{array}{l}\mathrm{n}=49 \text { Males: } 26 \\
\text { Age mean: } 68 \text { (range } 46- \\
88 \text { ) } \\
\text { Mixed population; chronic } \\
\text { obstructive pulmonary } \\
\text { disease }=28, \text { cancer }=9 \\
\text { heart disease }=5, \text { others } \\
=7 \\
\text { Baseline dyspnoea score } \\
\text { Mean numerical rating } \\
\text { scale }=5.7 ; 1.5\end{array}$ & $\begin{array}{l}\text { Hand-held } \\
\text { fan to face at } \\
\text { high or low } \\
\text { flow rate }\end{array}$ & $\begin{array}{l}\text { Usual care: } \\
\text { verbal and } \\
\text { written } \\
\text { exercise and } \\
\text { breathlessne } \\
\text { ss } \\
\text { management } \\
\text { advice }\end{array}$ & $\begin{array}{l}\text { Airflow } \\
\text { from } \\
\text { hand- } \\
\text { held } \\
\text { fan }\end{array}$ & $\begin{array}{l}\text { numerical } \\
\text { rating scale }\end{array}$ & $\begin{array}{l}\text { Withdrawals }=6 \\
\text { No Adverse events } \\
\text { Airflow preference: } \\
\text { positive patient } \\
\text { comments, numbers } \\
\text { not reported }\end{array}$ & $\begin{array}{l}\text { After } 4 \\
\text { weeks }\end{array}$ & $\begin{array}{l}\text { numerical rating scale }= \\
6.0 ; 2.0 \text { at } 4 \text { weeks } \\
\text { Mean change } 0.0 ; 3.0\end{array}$ & $\begin{array}{l}\text { No, phase II } \\
\text { not powered to } \\
\text { test }\end{array}$ \\
\hline $\begin{array}{l}\text { Wong } \\
\text { (2017) [44] }\end{array}$ & $\begin{array}{l}\text { Feasibility } \\
\text { phase II } \\
\text { randomised } \\
\text { controlled trial }\end{array}$ & $\begin{array}{l}\mathrm{n}=30 \text { Males: } 14 \\
\text { Age: not reported } \\
\text { Lung cancer }=13 \text {, other } \\
\text { cancers = } 17 \\
\text { Baseline dyspnoea score } \\
\text { Control group: numerical } \\
\text { rating scale mean } \\
5.6 ; 1.55 \\
\text { Intervention group: } \\
\text { numerical rating scale } \\
\text { mean } 6.13 ; 2.48\end{array}$ & $\begin{array}{l}\text { Table fan } \\
\text { with low flow } \\
\text { rate }\end{array}$ & $\begin{array}{l}\text { Placebo } \\
\text { accompanie } \\
\text { d by carer }\end{array}$ & $\begin{array}{l}\text { Airflow } \\
\text { from } \\
\text { table } \\
\text { fan to } \\
\text { face for } \\
5 \\
\text { minutes }\end{array}$ & $\begin{array}{l}\text { numerical } \\
\text { rating scale }\end{array}$ & $\begin{array}{l}\text { No withdrawals } \\
\text { Adverse events not } \\
\text { reported } \\
\text { Airflow preference: } \\
\text { mixed patient } \\
\text { comments, numbers } \\
\text { not reported }\end{array}$ & $\begin{array}{l}\text { After } 5 \\
\text { minutes } \\
\text { at rest }\end{array}$ & $\begin{array}{l}\text { numerical rating scale }= \\
4.60 \text { after } 5 \text { minutes fan to } \\
\text { face } \\
\text { Mean change }-1.53(1.06) \\
p<0.001\end{array}$ & Yes \\
\hline
\end{tabular}


Table 1 Characteristics of included studies (medical air)

\begin{tabular}{|c|c|c|c|c|c|c|c|c|c|c|}
\hline Study author & $\begin{array}{l}\text { Study } \\
\text { Design }\end{array}$ & $\begin{array}{l}\text { Population } \\
\text { (mean; standard deviation) }\end{array}$ & Intervention & Comparator & $\begin{array}{l}\text { Mode of } \\
\text { gas } \\
\text { delivery }\end{array}$ & $\begin{array}{l}\text { Dyspnea } \\
\text { Outcome } \\
\text { measure(s) }\end{array}$ & $\begin{array}{l}\text { Other Outcomes: } \\
\text { withdrawals, AE, } \\
\text { airflow } \\
\text { preferences }\end{array}$ & $\begin{array}{l}\text { Timing of } \\
\text { measurement }\end{array}$ & $\begin{array}{l}\text { Results airflow } \\
\text { arm only (before } \\
\text { and after } \\
\text { treatment) } \\
\text { (mean; standard } \\
\text { deviation) }\end{array}$ & $\begin{array}{l}\text { Improvement } \\
\text { with airflow } \\
\text { Yes/No }\end{array}$ \\
\hline $\begin{array}{l}\text { Abernethy } \\
\text { (2010) [29] }\end{array}$ & $\begin{array}{l}\text { Double-blind } \\
\text { randomised } \\
\text { controlled } \\
\text { trial }\end{array}$ & $\begin{array}{l}\mathrm{n}=239 \text { Males: } 63 \% \\
\text { Age mean: Air }=74 \mathrm{yrs} ; 10 \\
\text { Mixed: chronic obstructive } \\
\text { pulmonary disease }=152, \\
\text { Primary lung cancer }=33 \\
\text { Baseline dyspnoea score: Am } \\
\text { air }=4.6 ; 2.4 \\
\mathrm{Pm} \text { air }=4.7 ; 2.3\end{array}$ & Oxygen & $\begin{array}{l}\text { Room air via } \\
\text { concentrator }\end{array}$ & $\begin{array}{l}2 \mathrm{l} / \mathrm{min} \text { via } \\
\text { nasal } \\
\text { cannula for } \\
\text { at least } \\
15 \mathrm{hrs} \text { a } \\
\text { day (long } \\
\text { term } \\
\text { oxygen } \\
\text { therapy) }\end{array}$ & $\begin{array}{l}\text { numerical } \\
\text { rating scale } \\
1-10\end{array}$ & $\begin{array}{l}\text { Withdrawals = } 15 \\
\text { Few adverse } \\
\text { events, number } \\
\text { not reported } \\
\text { Side-effects } \\
\text { reported } \\
\text { Airflow } \\
\text { preferences not } \\
\text { reported, oxygen } \\
\text { only }\end{array}$ & $\begin{array}{l}\text { Am and pm } \\
\text { each day, } \\
\text { within } 30 \\
\text { minutes of } \\
\text { waking and } \\
\text { bedtime for } 7 \\
\text { days }\end{array}$ & $\begin{array}{l}\text { Am }=-0.7 \\
\text { numerical rating } \\
\text { scale point } \\
\text { change } \\
\mathrm{Pm}=-0.5 \\
\text { numerical rating } \\
\text { scale point } \\
\text { change, }(p=0.5)\end{array}$ & Yes \\
\hline $\begin{array}{l}\text { Booth (1996) } \\
\text { [32] }\end{array}$ & $\begin{array}{l}\text { Single-blind } \\
\text { cross-over } \\
\text { randomised } \\
\text { controlled } \\
\text { trial }\end{array}$ & $\begin{array}{l}\mathrm{n}=38 \text { Males: } 22 \\
\text { Age Median: } 71 \text { Range: } 54- \\
\text { 90yrs } \\
\text { Lung Cancer } 20, \text { chronic } \\
\text { obstructive pulmonary disease } \\
\text { 13, Cardiac } 4 \\
\text { Baseline dyspnoea score: } \\
\text { Visual analogue scale } 59 \mathrm{~mm}\end{array}$ & Oxygen & Cylinder air & $\begin{array}{l}41 / \text { minute } \\
\text { for } 15 \\
\text { minutes via } \\
\text { nasal } \\
\text { cannula }\end{array}$ & $\begin{array}{l}\text { Visual } \\
\text { analogue } \\
\text { scale }(\mathrm{mm}) \\
\text { Modified } \\
\text { Borg Scale }\end{array}$ & $\begin{array}{l}\text { No withdrawals } \\
\text { Adverse events } \\
\text { and airflow } \\
\text { preferences not } \\
\text { reported }\end{array}$ & $\begin{array}{l}\text { After } 15 \\
\text { minutes of } \\
\text { breathing } \\
\text { oxygen or air } \\
\text { at rest. }\end{array}$ & $\begin{array}{l}\text { Visual analogue } \\
\text { scale }=-11 \mathrm{~mm} \\
\text { change after air } \\
48 \mathrm{~mm}, p<0.001\end{array}$ & Yes \\
\hline
\end{tabular}




\begin{tabular}{|c|c|c|c|c|c|c|c|c|c|c|}
\hline $\begin{array}{l}\text { Eaton (2006) } \\
\text { [45] }\end{array}$ & $\begin{array}{l}\text { Double-blind } \\
\text { parallel } \\
\text { randomised } \\
\text { controlled } \\
\text { trial }\end{array}$ & $\begin{array}{l}\mathrm{n}=78 \text { Males: } 36 \\
\text { Age mean: } 77.3 y r s ; 7.06 \\
\text { Moderate/severe chronic } \\
\text { obstructive pulmonary disease } \\
\text { Baseline chronic respiratory } \\
\text { questionnaire score: Air = } \\
17.5 ; 4.2\end{array}$ & Oxygen & Cylinder air & $\begin{array}{l}21 / \text { minute } \\
\text { via nasal } \\
\text { cannula } \\
\text { over } 6 \\
\text { months } \\
\text { (SBOT) }\end{array}$ & CRQ & $\begin{array}{l}\text { Withdrawals = } 21 \\
\text { Adverse events } \\
\text { and airflow } \\
\text { preferences not } \\
\text { reported }\end{array}$ & $\begin{array}{l}\text { Monthly over } 6 \\
\text { months }\end{array}$ & $\begin{array}{l}\text { chronic respiratory } \\
\text { questionnaire = } \\
\text { Average change } \\
\text { over } 6 \text { months: air } \\
\text { group }=-3.6\end{array}$ & No \\
\hline $\begin{array}{l}\text { Eves (2009) } \\
{[46]}\end{array}$ & $\begin{array}{l}\text { Double-blind } \\
\text { randomised } \\
\text { controlled } \\
\text { trial }\end{array}$ & $\begin{array}{l}\mathrm{n}=38 \text { Males: } 23 \\
\text { Age mean: } 65.5 \mathrm{yrs} \text { (SD 8) } \\
\text { Stable chronic obstructive } \\
\text { pulmonary disease } \\
\text { Baseline dyspnoea score: } \\
\text { constant load exercise Borg } \\
\text { mean: Air = 6.0; } 2.2 \\
\text { incremental load exercise Borg } \\
\text { mean: Air = 5.6; } 2.0\end{array}$ & $\begin{array}{l}\text { Helium- } \\
\text { hyperoxia } \\
(60 \% \mathrm{HE} \text { : } \\
\left.40 \% \mathrm{O}^{2}\right)\end{array}$ & Cylinder air & $\begin{array}{l}\text { Face mask } \\
\text { (non- } \\
\text { rebreathing } \\
\text { ) }\end{array}$ & $\begin{array}{l}\text { Modified } \\
\text { Borg score }\end{array}$ & $\begin{array}{l}\text { No withdrawals } \\
\text { No Adverse } \\
\text { events } \\
\text { Airflow } \\
\text { preferences not } \\
\text { reported }\end{array}$ & $\begin{array}{l}\text { During } \\
\text { exercise test } \\
\text { before and } \\
\text { after } 6 \text { weeks } \\
\text { pulmonary } \\
\text { rehabilitation } \\
\text { programme, } 3 \\
\text { times a week } \\
\text { for } 30 \text { minutes } \\
\text { on cycle } \\
\text { ergometer }\end{array}$ & $\begin{array}{l}\text { constant load } \\
\text { exercise Borg } \\
\text { mean: Air = 4.2; } \\
2.1 \text { mean change } \\
=-1.8(95 \% \mathrm{Cl}- \\
3.1 \text { to }-0.2), \mathrm{p}< \\
0.05 \\
\text { incremental load } \\
\text { exercise Borg } \\
\text { mean: Air = 5.6; } \\
2.1 \\
\mathrm{No} \text { change }(95 \% \\
\mathrm{Cl}-0.7 \text { to } 0.7)\end{array}$ & Yes \\
\hline $\begin{array}{l}\text { Jolly (2001) } \\
\text { [47] }\end{array}$ & $\begin{array}{l}\text { Double-blind } \\
\text { randomised } \\
\text { controlled } \\
\text { trial }\end{array}$ & $\begin{array}{l}\mathrm{n}=20 \text { Males: } 19 \\
\text { Age mean: 68.5yrs (SEM 2.5) } \\
\text { Stable chronic obstructive } \\
\text { pulmonary disease } \\
\text { Baseline dyspnoea score: } \\
\text { Borg mean score } \\
\text { Desat group Baseline 6minute } \\
\text { walk test = 5.82 (SEM 0.46) } \\
\text { Non-desat group Baseline } \\
\text { 6minute walk test = } 4.22 \text { (SEM } \\
0.46 \text { ) }\end{array}$ & Oxygen & Cylinder air & $\begin{array}{l}31 / \text { minute } \\
\text { via nasal } \\
\text { cannula }\end{array}$ & $\begin{array}{l}\text { Modified } \\
\text { Borg score }\end{array}$ & $\begin{array}{l}\text { No withdrawals } \\
\text { Adverse events } \\
\text { and airflow } \\
\text { preferences not } \\
\text { reported }\end{array}$ & $\begin{array}{l}\text { Before and } \\
\text { after } 3 \times 6 \\
\text { MWTs with at } \\
\text { least } \\
45 \text { minutes } \\
\text { washout } \\
\text { between walks }\end{array}$ & $\begin{array}{l}\text { Borg mean score: } \\
\text { Desat group } \\
\text { Air 6minute walk } \\
\text { test = } 5.82 \text { (SEM } \\
0.42 \text { ) } \\
\text { No change } \\
\text { Non-desat group } \\
\text { Air 6minute walk } \\
\text { test = } 4.44 \text { (SEM } \\
0.73 \text { ) } \\
\text { No change }\end{array}$ & No \\
\hline
\end{tabular}




\begin{tabular}{|c|c|c|c|c|c|c|c|c|c|c|}
\hline $\begin{array}{l}\text { Marciniuk } \\
(2007) \text { [48] }\end{array}$ & $\begin{array}{l}\text { Double-blind } \\
\text { crossover } \\
\text { randomised } \\
\text { controlled } \\
\text { trial }\end{array}$ & $\begin{array}{l}\mathrm{n}=16 \text { Males: } 7 \\
\text { Age mean: } 67 \text { (SD 8) } \\
\text { Moderate to severe chronic } \\
\text { obstructive pulmonary disease } \\
\text { Baseline dyspnoea score: } \\
\text { Borg mean score Baseline } \\
6 \text { minute walk test }=5 ; 2\end{array}$ & $\begin{array}{l}100 \% \\
\text { Oxygen or } \\
\text { Helium- } \\
\text { hyperoxia } \\
(70 \% \mathrm{HE}: \\
\left.30 \% \mathrm{O}^{2}\right)\end{array}$ & Cylinder air & $\begin{array}{l}15 \mathrm{l} / \text { minute } \\
\text { via face } \\
\text { mask } \\
81 / \text { minute } \\
\text { via nasal } \\
\text { cannula }\end{array}$ & $\begin{array}{l}\text { Modified } \\
\text { Borg score }\end{array}$ & $\begin{array}{l}\text { No withdrawals } \\
\text { Adverse events } \\
\text { and airflow } \\
\text { preferences not } \\
\text { reported }\end{array}$ & $\begin{array}{l}\text { Before and } \\
\text { after each } 6 \\
\text { MWTs on visit } \\
1,2 \text { and } 3 \text { with } \\
60 \text { minutes } \\
\text { washout } \\
\text { between walks }\end{array}$ & $\begin{array}{l}\text { Borg mean score } \\
\text { After 6MWT Air = } \\
3.5 \\
\text { mean Borg score } \\
\text { change }=-1.5 \\
\text { decrease }\end{array}$ & Yes \\
\hline $\begin{array}{l}\text { McDonald } \\
\text { (1995) [49] }\end{array}$ & $\begin{array}{l}\text { Double-blind } \\
\text { crossover } \\
\text { randomised } \\
\text { controlled } \\
\text { trial }\end{array}$ & $\begin{array}{l}\mathrm{n}=26 \text { Males: } 24 \\
\text { Age mean: } 73 ; 6 \\
\text { Stable severe chronic } \\
\text { obstructive pulmonary disease } \\
\text { Baseline dyspnoea score } \\
6 \text { minute walk test : Air group = } \\
3.8 ; 1.4 \text { chronic respiratory } \\
\text { questionnaire }=14 ; 5\end{array}$ & Oxygen & Cylinder air & $\begin{array}{l}41 / \text { minute } \\
\text { via nasal } \\
\text { cannula }\end{array}$ & $\begin{array}{l}\text { Modified } \\
\text { Borg score } \\
\text { chronic } \\
\text { respiratory } \\
\text { questionnai } \\
\text { re }\end{array}$ & $\begin{array}{l}\text { Withdrawals = } 7 \\
\text { Adverse events } \\
\text { and airflow } \\
\text { preferences not } \\
\text { reported }\end{array}$ & $\begin{array}{l}\text { After } 6 \text { and } 12 \\
\text { weeks of } \\
\text { home cylinder } \\
\text { air using } \\
6 \mathrm{MWT} \\
\text { exercise test } \\
\text { with } 20 \text { minute } \\
\text { washout } \\
\text { between walks }\end{array}$ & $\begin{array}{l}\text { Borg Mean score } \\
\text { Home air: 6minute } \\
\text { walk test with } \\
\text { cylinder air = } 3.8 \\
\text { (SD 1.5) No } \\
\text { change } \\
\text { chronic respiratory } \\
\text { questionnaire } \\
\text { score Home air = } \\
17 ; 6 \\
3 \text { point change }\end{array}$ & $\begin{array}{l}\text { No with } \\
6 \text { minute walk } \\
\text { test } \\
\text { Yes with } \\
\text { chronic } \\
\text { respiratory } \\
\text { questionnaire }\end{array}$ \\
\hline $\begin{array}{l}\text { Moore } \\
\text { (2011) [50] }\end{array}$ & $\begin{array}{l}\text { Double-blind } \\
\text { randomised } \\
\text { controlled } \\
\text { trial }\end{array}$ & $\begin{array}{l}\mathrm{n}=143 \text { Males: } 99 \\
\text { Age mean: } 71.8 y r s ; 9.8 \\
\text { Range: } 43-78 \\
\text { Stable chronic obstructive } \\
\text { pulmonary disease } \\
\text { Baseline dyspnoea score: Air } \\
=17.5 ; 4.9\end{array}$ & Oxygen & Cylinder air & $\begin{array}{l}61 / \text { minute } \\
\text { via nasal } \\
\text { cannula at } \\
\text { home for } \\
12 \text { weeks } \\
\text { with activity } \\
\text { (SBOT) }\end{array}$ & $\begin{array}{l}\text { chronic } \\
\text { respiratory } \\
\text { questionnai } \\
\text { re }\end{array}$ & $\begin{array}{l}\text { Withdrawals = } 4 \\
\text { Adverse events } \\
\text { not reported } \\
\text { Airflow } \\
\text { preferences } 45 \% \\
\text { prefer no cylinder }\end{array}$ & $\begin{array}{l}\text { At } 4 \text { weeks } \\
\text { and } 12 \text { weeks }\end{array}$ & $\begin{array}{l}\text { Air: } 4 \text { weeks = } \\
18.4 ; 5.8 \\
12 \text { weeks = 18.4; } \\
5.8 \\
\text { Air: chronic } \\
\text { respiratory } \\
\text { questionnaire = } \\
\text { Mean change at } 4 \\
\text { and } 12 \text { weeks = } \\
0.9\end{array}$ & Yes \\
\hline $\begin{array}{l}\text { Philip (2006) } \\
\text { [51] }\end{array}$ & $\begin{array}{l}\text { Double-blind } \\
\text { cross-over } \\
\text { randomised } \\
\text { controlled } \\
\text { trial }\end{array}$ & $\begin{array}{l}\mathrm{n}=51 \text { Males: } 31 \\
\text { Age median: } 65 \text { Range: } 33- \\
\text { 82yrs } \\
\text { Non small cell lung cancer }= \\
22, \text { Small cell lung cancer }=6 \text {, } \\
\text { Breast }=8, \text { Colorectal }=4 \\
\text { Others }=11\end{array}$ & Oxygen & Medical Air & $\begin{array}{l}41 / \text { minute } \\
\text { for } 15 \\
\text { minutes via } \\
\text { nasal } \\
\text { cannula }\end{array}$ & $\begin{array}{l}\text { Visual } \\
\text { analogue } \\
\text { scale (mm) }\end{array}$ & $\begin{array}{l}\text { No withdrawals } \\
\text { Adverse events } \\
\text { not reported } \\
\text { Airflow } \\
\text { preferences: } \\
\text { Positive: } \mathrm{n}=15 \\
(29 \%)\end{array}$ & $\begin{array}{l}\text { Before and } \\
\text { after } 15 \\
\text { minutes of gas }\end{array}$ & $\begin{array}{l}\text { Visual analogue } \\
\text { scale median } \\
\text { After air } 1 \text { st = } \\
\text {-3mm change } \\
\text { (range -19 to } 7 \text { ) }\end{array}$ & Yes \\
\hline
\end{tabular}




\begin{tabular}{|c|c|c|c|c|c|c|c|c|c|c|}
\hline & & $\begin{array}{l}\text { Baseline dyspnoea score: } \\
\text { Visual analogue scale median } \\
\text { Air } 1 \mathrm{st}=52 \mathrm{~mm} \text { (range } 23-92) \\
\text { Visual analogue scale median } \\
\text { Air } 2 \mathrm{nd}=42 \mathrm{~mm} \text { (range 10-70) }\end{array}$ & & & & & & & $\begin{array}{l}\text { Visual analogue } \\
\text { scale median } \\
\text { After air } 2 \mathrm{nd}= \\
-11.5 \mathrm{~mm} \text { change } \\
\text { (range }-20 \text { to } 45 \text { ) } \\
\text { Visual analogue } \\
\text { scale mean } \\
\text { change }=- \\
13.4 \mathrm{~mm}\end{array}$ & \\
\hline $\begin{array}{l}\text { Scorsone } \\
(2010) \text { [52] }\end{array}$ & $\begin{array}{l}\text { Double-blind } \\
\text { randomised } \\
\text { controlled } \\
\text { trial }\end{array}$ & $\begin{array}{l}\mathrm{n}=30 \text { Males: } 23 \\
\text { Age mean: } 67.3 y r s \text { (SD } 8.3 \text { ) } \\
\text { Moderate to severe chronic } \\
\text { obstructive pulmonary disease } \\
\text { Baseline dyspnoea score: } \\
\text { Before training incremental } \\
\text { load exercise Borg: Air }=7 ; 3 \\
\text { Before training constant load } \\
\text { exercise Borg: Air }=8 ; 3\end{array}$ & $\begin{array}{l}40 \% \text { Oxygen } \\
\text { or Helium - } \\
\text { hyperoxia } \\
(60 \% \mathrm{HE}: \\
\left.40 \% \mathrm{O}^{2}\right)\end{array}$ & $\begin{array}{l}\text { Humidified } \\
\text { room air }\end{array}$ & $\begin{array}{l}\text { Mouthpiec } \\
\text { e from a } \\
\text { Douglas } \\
\text { bag }\end{array}$ & $\begin{array}{l}\text { Modified } \\
\text { Borg score }\end{array}$ & $\begin{array}{l}\text { No withdrawals } \\
\text { Adverse events } \\
\text { and airflow } \\
\text { preferences not } \\
\text { reported }\end{array}$ & $\begin{array}{l}\text { During } \\
\text { exercise } \\
\text { before and } \\
\text { after a } 2 \\
\text { months } \\
\text { pulmonary } \\
\text { rehabilitation } \\
\text { programme, } 3 \\
\text { times a week } \\
\text { for } 20 \text { minutes } \\
\text { on cycle } \\
\text { ergometer }\end{array}$ & $\begin{array}{l}\text { After training } \\
\text { incremental load } \\
\text { exercise Borg: Air } \\
=4 ; 2 \\
\text { After training } \\
\text { constant load } \\
\text { exercise Borg = } 5 \text {; } \\
3 \\
\text { Borg change = -3 } \\
\text { point decrease } \\
\text { both exercise } \\
\text { tests }\end{array}$ & Yes \\
\hline $\begin{array}{l}\text { Wadell } \\
\text { (2001) [53] }\end{array}$ & $\begin{array}{l}\text { Single-blind } \\
\text { crossover } \\
\text { randomised } \\
\text { controlled } \\
\text { trial }\end{array}$ & $\begin{array}{l}n=20 \text { Males: } 10 \\
\text { Age mean: 67yrs Range: } 52- \\
73 \\
\text { Stable chronic obstructive } \\
\text { pulmonary disease } \\
\text { Baseline dyspnoea median } \\
\text { score: } \\
\text { Test A (Air) At rest; Pre- } \\
\text { training Borg: Air group = } 1.5 \\
\text { (0-3) } \\
\text { Test A (Air) After 6minute walk } \\
\text { test, Pre-training Borg: Air } \\
\text { group = 6.5 (4-9) }\end{array}$ & Oxygen & Air & $\begin{array}{l}51 / \text { minute } \\
\text { via nasal } \\
\text { cannula }\end{array}$ & $\begin{array}{l}\text { Modified } \\
\text { Borg score }\end{array}$ & $\begin{array}{l}\text { Withdrawals }=2 \\
\text { Adverse events } \\
\text { and patient } \\
\text { preferences not } \\
\text { reported }\end{array}$ & $\begin{array}{l}\text { During } \\
\text { exercise using } \\
2 \times 6 \text { minute } \\
\text { walk test } \\
\text { (air/O² or } \\
\mathrm{O}^{2} / \text { air) with } \\
\text { 1hour washout } \\
\text { before and } \\
\text { after a } 2 \\
\text { months } \\
\text { pulmonary } \\
\text { rehabilitation } \\
\text { programme, } 3 \\
\text { times a week } \\
\text { for } 30 \text { minutes } \\
\text { on a treadmill }\end{array}$ & $\begin{array}{l}\text { Test A (Air) At } \\
\text { rest; Post-training } \\
\text { Borg: Air group = } \\
1(0-3) \\
\text { Test A (Air) After } \\
6 \text { minute walk test } \\
\text {, Post-training } \\
\text { Borg: Air group = } \\
6(1-7) \\
\text { Borg change = } \\
-0.5 \text { point at rest } \\
\text { and after exercise } \\
\text { test }\end{array}$ & Yes \\
\hline
\end{tabular}




\section{Risk of Bias}

The quality appraisal is summarised in Online Supplementary Table 2 and described below.

Allocation: all of the studies, apart from one, a cohort design (14), were described as randomised controlled trials. It was possible to verify the randomisation process in eight studies. $(13,16,17,29,32,45,46,50)$. There was insufficient information to determine the risk of allocation bias in the other randomised controlled trials. (44, 47-49, 51-53)

Blinding: two of the fan studies attempted to blind the participants $(16,17)$; a placebo wristband was used as a comparator (16) and participants were not told if the fan to face or fan to leg was the active intervention. (17) There was no blinding in two studies, a cohort and phase II randomised controlled trial $(13,14)$, and the fifth study stated single blinding that could not be verified from the methods described. (44) All five were judged high risk of bias due to incomplete blinding or limited description. Nine medical air randomised controlled trials were described as double blind. (29, 45-52) All were judged low risk of bias (29, 45, 46, 48-50, 52), apart from one study that was unclear due to the lack of detail reported. (51) Two randomised controlled trials were single blind $(32,53)$; one was judged low risk of bias (32) and the other was regarded as unclear risk due to the inadequate description. (53)

Incomplete outcome data: 13 studies adequately addressed withdrawals and incomplete outcome data; these were considered low risk of bias.(13, 14, 17, 29, 32, 46-53) Three studies were uncertain risk $(16,45)$; one due to the proportion of attrition (16) and the other two lacked description of how any missing data were statistically managed. $(44,45)$

Selective Outcome reporting: all of the studies reported the pre-specified outcomes and were judged as low risk of bias. $(13,14,16,17,29,32,44-53)$ Study protocols were available for eight studies. (13, 14, 16, 17, 29, 46, 50, 51)

Other issues of bias: twelve studies appeared free from other bias and were judged low risk. $(13,16,17,29,44-46,48,50-53)$ Three studies reported insufficient information to adequately assess risk (32, 47, 49), and one study, a cohort design was judged high risk. (14) 


\section{Effect of interventions}

The airflow was delivered, i) at rest $(14,17,32,44,51)$ ii) over days or weeks (either intermittently or as periods of continuous flow) whilst the participant continued with usual general activities $(13,16,29,45,50)$ or iii) during specific episodes of exertion induced breathlessness. (46-49, 52, 53)

\section{i) At rest}

Five studies demonstrated improvement with airflow delivery at rest.

Results from 5 minutes fan use to the face in three studies were visual analogue scale breathlessness intensity difference from baseline mean -7mm (CI -11.5 to -2.5) (17), and mean $-12 \mathrm{~mm}$ (CI -19.3 to -4.4$)(14)$, and for the numerical rating scale mean change -1.53 (9.6 to -6.5$).(44)$

Cylinder medical air delivery for 15 minutes demonstrated improvement visual analogue scale breathlessness intensity mean $-11 \mathrm{~mm}$ (CI -17.0 to -5.0 ) (32), and mean $-13 \mathrm{~mm}$ (CI 20.5 to-6.3). (51) Four studies were sub-divided into two groups and included in metaanalyses.

\section{Fan}

Airflow from the fan at rest improved breathlessness in a mixed population ( $\mathrm{n}=111 ; 58 \%$ cancer) visual analogue scale ( $\mathrm{mm}$ ) mean difference, -11.17 (confidence intervals -16.60 to 5.74), $\mathrm{p}=0.06$. Significant heterogeneity was observed, $\mathrm{Chi}^{2} \mathrm{p}$-value $=0.2$, $\left(\mathrm{I}^{2}=64 \%\right)$ (See Figure 2).

\section{$<<$ insert Figure 2 Meta-analysis of fan at rest $>>$}

\section{Medical air}

Airflow delivered as cylinder medical air at rest improved breathlessness in advanced cancer $(\mathrm{n}=89)$ visual analogue scale $(\mathrm{mm})$ mean difference -12.0, (confidence intervals -16.6 to 7.4), $\mathrm{P}<0.0001$. No evidence of heterogeneity was observed, $\mathrm{Chi}^{2} \mathrm{P}$ value $=0.6,\left(\mathrm{I}^{2}=0 \%\right)$. 


\section{$<<$ insert Figure 3 Meta-analysis of cylinder air at rest $>>$}

\section{ii) General activity}

Six studies used airflow at home with everyday general activity. A narrative description was used for these due to study diversity. Breathlessness points change from four cylinder air studies were mixed (29, 45, 49, 50), with Chronic Respiratory Questionnaire -3.6 after 6 months (45), 3.0 after 12 weeks (49), or 0.9 at 12 weeks (50), or numerical rating scale -0.7 (am) and -0.5 numerical rating scale (pm) after 7 days. (29) In the two fan studies a modified Borg score of -0.6 (SD 2.1) was found after 2 months (16), but there was no numerical rating scale score change after 4 weeks of fan use with exercise advice. (13)

\section{iii) Exertion-induced breathlessness}

Six studies examined airflow delivery with exertion-induced breathlessness. Results for mean Borg breathlessness score during a walking test for three studies varied; no change during a 6 minute walk test repeated on the same day (47), or at 12 weeks (49), and improvement -1.5 for a 6 minute walk test repeated on 3 separate visits. (48) Airflow delivered during a constant load exercise test after PR in three studies also demonstrated variable improvement in mean Borg breathlessness scores; -1.8 points (46), and -3 point (52) using a cycle ergometer, and - 0.5 point from a treadmill test. (53) Two studies were suitable to include in a meta-analysis (See Figure 4). (46, 52)

\section{Medical air}

Airflow delivered as cylinder medical air during a constant load exercise test after PR in chronic obstructive pulmonary disease $(\mathrm{n}=29)$ significantly improved breathlessness Borg score mean difference -2.9, (CI -3.2 to -2.7), $\mathrm{p}<0.0001$. No evidence of heterogeneity was observed, $\mathrm{Chi}^{2} \mathrm{p}$-value $=0.7,\left(\mathrm{I}^{2}=0 \%\right)$, Figure 4$)$.

\section{$<<$ insert Figure 4 Meta-analysis of cylinder medical air for exertion-induced breathlessness $>>$}




\section{Discussion}

These exploratory data support that facial and nasal airflow delivery at rest offers relief of breathlessness intensity consistent with a moderate clinically important difference, $(54,55)$ and during exertion. $(46,52)$ All participants in the cylinder medical air delivery at rest studies had advanced cancer, but nearly half of those in the fan "at rest” studies had other conditions indicating that airflow for breathlessness at rest is of benefit irrespective of cause.

In a recent pooled qualitative data study of facial airflow use from the fan in 133 people with chronic breathlessness (56), over 80\% patients reported some or substantial benefit.(57) However, the data presented here varied with regard to relief of breathlessness intensity when facial or nasal airflow delivery was used with everyday general activity or with exertion induced breathlessness. This may reflect the use of outcome measures that do not reliably capture change in breathlessness intensity in the context of exertion. Studies that used a 6 minute walk test (47-49) highlight the problem of a self-paced test that allows patients to control their walking speed and thus limit the maximal level of exertion-induced breathlessness experienced. In contrast, studies that used an externally paced test, such as the cycle ergometer, identified relief of breathlessness intensity. $(46,52)$ The relationship between exercise and breathlessness intensity is complex, and measuring one without taking the other into account may miss relevant improvement. Scores are likely to remain static after the introduction of an intervention as patients are able to exert themselves to the same level of breathlessness without noticing an increase in their exercise tolerance (58), or indeed the outcome may be of little value to the patient. (57)

A previous study of recovery time after an incremental shuttle walk test in people with thoracic cancer $(n=57)$ reported a rapid reduction in breathlessness intensity with a return to baseline time of median 4 (interquartile range 2-5) minutes. (59) The analysis of 133 patient interviews found that a faster recovery time was a key patient-reported benefit of airflow delivered from the fan, irrespective of breathlessness intensity. (57) Even though recovery time may only be a matter of minutes, interventions which shorten this further are clearly welcomed and give the patient a sense of self-control that may help prevent a breathlessnessanxiety spiral. The ability to recover quickly and predictably from bouts of exertion is likely to encourage further activity and prevent the deconditioning cycle. 
The fan therefore seems suitable as a patient-delivered intervention to target the recovery time from exertion-induced breathlessness. Preliminary magnetoencephalography imaging data suggests airflow delivery during recovery from exercise may modulate central perception of breathlessness by modifying sensory attention. (60) Cooling of the facial skin innervated by the $2^{\text {nd }}$ and $3^{\text {rd }}$ branches of the trigeminal nerve and/or stimulation of nasal mucosa and upper airway 'flow' receptors are reported to improve breathlessness intensity and exercise tolerance $(18,19,61,62)$ and could "fool" the brain into thinking that the respiratory status is adequate. (22)

Unpleasant respiratory sensations associated with exercise are known to adversely influence adherence to an exercise regime. (63) Therefore, use of airflow as part of pulmonary rehabilitation may help the problems of low patient attendance and poor maintenance of long term outcomes. (64-67) Facial airflow from fan use during a cycle ergometer test in chronic obstructive pulmonary disease patients resulted in significant breathlessness reduction and a longer total exercise time. (68) Likewise, the meta-analysis result from this systematic review suggest significant relief of breathlessness when airflow is delivered during exercise. These data highlight the potential value of using airflow delivery with pulmonary rehabilitation or home based exercise programmes. In addition, intervention preference and adverse event data support the role of the fan in this context as a portable device that is unlikely to harm and therefore appropriate for the majority of patients to try.

Finally, it is likely that any positive benefits of airflow delivery from fan use with everyday general activity and at rest were not captured in the review data. The lack of signal from the results may in part reflect the complexity and the nuances of when, where and how this intervention is used by patients. (57) Current breathlessness management is modelled on a complex intervention, of which the fan is identified as a valuable therapeutic component alongside other interventions and strategies that are tailored to the patient's breathlessness needs. $(11,69)$

\section{Limitation of methods}

Data were analysed as cohort "before and after" design, and no adjustments were made to control for confounding bias. The pre-post comparison increases the potential risk of bias and it is possible that results may be influenced by the timing of "before and after" measures. For 
example, studies of longer duration (up to 6 months) may not be representative of the immediate benefits of airflow, but rather reflect more complex use and mechanism of any observed benefit may be related to reconditioning, facilitated by airflow, over time. Risk of bias was assessed using a tool designed for randomised controlled trials therefore it is possible that this assessment may not capture potential sources of bias associated with the observational methods used in this systematic review.

Overall, the qualitative synthesis represents findings from 929 participants the largest to date, however the meta-analyses pertain to a small number of participants and only provide a preliminary indication of the pooled effect estimate of airflow. The meta-analyses involve few studies therefore heterogeneity is difficult to estimate and the accuracy of the $\mathrm{I}^{2}$ value is less certain. (70) The number of studies that fulfilled the review criteria was restricted by the need for baseline breathlessness measures. Some of the included studies $(32,51)$ did not report repeated measurements in a format suitable for meta-analysis necessitating statistical assumptions. (42)

\section{Implications for practice and further research}

Airflow is safe and should be used as an adjunct to treatment for breathlessness at rest in those who do not require oxygen-enriched air. Clinicians should consider airflow an important intervention to use as part of a breathlessness management programme in breathlessness at rest irrespective of cause. The relief of breathlessness during exertion in those with chronic obstructive pulmonary disease may provide a useful intervention during pulmonary rehabilitation where breathlessness is a reason for poor adherence.

The fan, when taught by an appropriately trained clinician, offers patients an inexpensive and portable source of airflow likely to benefit exertion-induced breathlessness. Recovery time from exertion induced breathlessness is an important patient-reported outcome and further work is needed to explore the role of airflow in recovery, self-efficacy and increased daily activity as part of complex breathlessness intervention programmes including rehabilitation. 


\section{Conclusion}

These data support facial or nasal airflow for clinically meaningful relief of breathlessness at rest. This systematic review pulls together the growing evidence to support airflow as an effective self-management option for people with chronic breathlessness and identifies airflow as an intervention for future study.

\section{Declarations.}

Authorship: Concept - FS; Design - FS, MJJ, CB, SB, JY; Data collection - FS, AN; Data analysis - FS, VA, MB; Data interpretation- All; Draft manuscript FS; critical revision of manuscript for intellectual content - All; approval final manuscript - All.

Funding: This study was funded as part of a University of Hull PhD studentship (Flavia Swan)

Declaration of conflicts of interest: The Authors declare that there is no conflict of interest.

Data management and sharing: The full search strategy is found in the Online Supplementary materials and included and excluded papers are presented. 


\section{References}

1. Currow DC, Dal Grande E, Ferreira D, Johnson MJ, McCaffrey N, Ekström M. Chronic breathlessness associated with poorer physical and mental health-related quality of life (SF-12) across all adult age groups. Thorax. 2017.

2. Smith AK, Currow DC, Abernethy AP, Johnson MJ, Miao Y, Boscardin WJ, et al. Prevalence and Outcomes of Breathlessness in Older Adults: A National Population Study. Journal of the American Geriatrics Society. 2016;64(10):2035-41.

3. Parshall MB. Adult Emergency Visits for Chronic Cardiorespiratory Disease: Does Dyspnea Matter? Nurs Res. 1999;48(2):62-70.

4. Parshall MB, Doherty GS. Predictors of emergency department visit disposition for patients with chronic obstructive pulmonary disease. Heart \& Lung: The Journal of Acute and Critical Care.35(5):342-50.

5. Hutchinson A, Pickering A, Williams P, Bland JM, Johnson MJ. Breathlessness and presentation to the emergency department: a survey and clinical record review. BMC Pulmonary Medicine. 2017;17:1-7.

6. Bausewein C, Booth S, Gysels M, Kuhnbach R, Haberland B, Higginson IJ. Understanding breathlessness: cross-sectional comparison of symptom burden and palliative care needs in chronic obstructive pulmonary disease and cancer. Journal of Palliative Medicine. 2010;13(9):1109-18.

7. Booth S, Silvester S, Todd C. Breathlessness in cancer and chronic obstructive pulmonary disease: using a qualitative approach to describe the experience of patients and carers. Palliative \& Supportive Care. 2003;1(4):337-44.

8. Williams V, Bruton A, Ellis-Hill C, McPherson K. What really matters to patients living with chronic obstructive pulmonary disease? An exploratory study. Chronic Respiratory Disease. 2007;4(2):77-85.

9. Johnson MJ, Yorke J, Hansen-Flaschen J, Lansing R, Ekström M, Similowski T, et al. Towards an expert consensus to delineate a clinical syndrome of chronic breathlessness. European Respiratory Journal. 2017;49(5).

10. Farquhar MC, Prevost AT, McCrone P, Brafman-Price B, Bentley A, Higginson IJ, et al. The clinical and cost effectiveness of a Breathlessness Intervention Service for patients with advanced non-malignant disease and their informal carers: mixed findings of a mixed method randomised controlled trial. Trials. 2016;17(1):1-16.

11. Higginson IJ, Bausewein C, Reilly CC, Gao W, Gysels M, Dzingina M, et al. An integrated palliative and respiratory care service for patients with advanced disease and refractory breathlessness: a randomised controlled trial. The Lancet Respiratory Medicine. 2014;2(12):979-87. 12. Barton R EA, Nabb S, Rigby AS, and Johnson MJ A randomised trial of high Vs low intensity training in breathing techniques for breathless patients with malignant lung disease: A feasibility study. Lung cancer 2010;70:313-9.

13. Johnson MJ, Booth S, Currow DC, Lam LT, Phillips JL. A Mixed-Methods, Randomized, Controlled Feasibility Trial to Inform the Design of a Phase III Trial to Test the Effect of the Handheld Fan on Physical Activity and Carer Anxiety in Patients With Refractory Breathlessness. Journal of Pain and Symptom Management. 2016;51(5):807-15.

14. Booth S, Galbraith S, Ryan R, Parker RA, Johnson M. The importance of the feasibility study: Lessons from a study of the hand-held fan used to relieve dyspnea in people who are breathless at rest. Palliative Medicine. 2016;30(5):504-9.

15. Swan F BS. The role of airflow for the relief of chronic refractory breathlessness. . Current Opinion in Supportive \& Palliative Care 2015;Sept 9(3):206-11.

16. Bausewein C BS, Gysels M, Kuhnbach R, and Higginson I J,. Effectiveness of a hand-held fan for breathlessness: a randomised phase II trial. BMC Palliative Care 2010;9(22). 
17. Galbraith S, Fagan P, Perkins P, Lynch A, Booth S. Does the use of a handheld fan improve chronic dyspnea? A randomized, controlled, crossover trial. Journal of Pain and Symptom Management [Internet]. 2010; (5):[831-8 pp.].

18. Schwartzstein RM, Lahive K, Pope A, Weinberger SE, Weiss JW. Cold Facial Stimulation Reduces Breathlessness Induced in Normal Subjects. American Journal of Respiratory and Critical Care Medicine. 1987;136(1):58-61.

19. Liss HP GJ. The effect of nasal flow on breathlessness in patients with chronic obstructive pulmonary disease. American Review Respiratory disease 1988;137:1285-8.

20. Parshall MB, Schwartzstein RM, Adams L, Banzett RB, Manning HL, Bourbeau J, et al. An Official American Thoracic Society Statement: Update on the Mechanisms, Assessment, and Management of Dyspnea. American Journal of Respiratory and Critical Care Medicine. 2012;185(4):435-52.

21. Johnson MJ, Simpson MIG, Currow DC, Millman RE, Hart SP, Green G.

Magnetoencephalography to investigate central perception of exercise-induced breathlessness in people with chronic lung disease: a feasibility pilot. BMJ Open. 2015;5(6):e007535.

22. Morélot-Panzini C. Fooling the brain to alleviate dyspnoea. European Respiratory Journal. 2017;50(2).

23. Luckett T PJ, Johnson MJ, Farquhar M, Swan F, Assen T, Bhattarai P, Booth S. . Contributions of a hand-held fan to self-management of chronic breathlessness. . European Respiratory Journal. 2017;50(1700262).

24. Mularski R, Reinke L, al C-KVe. An official American Thoracic Society workshop report: Assessment and palliative management of dyspnea crisis. Annals American Thoracic Society. 2013;10(5):S98-105.

25. Ben-Aharon I, Gafter-Gvili A, Paul M, Leibovici L, Stemmer SM. Interventions for Alleviating Cancer-Related Dyspnea: A Systematic Review. Journal of Clinical Oncology. 2008;26(14):2396-404. 26. Booth S, Wade R, Johnson M, Kite S, Swannick M, Anderson $\mathrm{H}$, et al. The use of oxygen in the palliation of breathlessness. A report of the expert working group of the Scientific Committee of the Association of Palliative Medicine. Respiratory Medicine. 2004;98(1):66-77.

27. Cranston Josephine $M$, Crockett A, Currow D. Oxygen therapy for dyspnoea in adults. Cochrane Database of Systematic Reviews [Internet]. 2008; (3).

28. Uronis HE, Currow DC, McCrory DC, Samsa GP, Abernethy AP. Oxygen for relief of dyspnoea in mildly- or non-hypoxaemic patients with cancer: a systematic review and meta-analysis. $\mathrm{Br} \mathrm{J}$ Cancer. 2008;98(2):294-9.

29. Abernethy AP, McDonald CF, Frith PA, Clark K, Herndon JE, 2nd, Marcello J, et al. Effect of palliative oxygen versus room air in relief of breathlessness in patients with refractory dyspnoea: a double-blind, randomised controlled trial. Lancet. 2010;376(9743):784-93.

30. Bell EC, Cox NS, Goh N, Glaspole I, Westall GP, Watson A, et al. Oxygen therapy for interstitial lung disease: a systematic review. European Respiratory Review. 2017;26(143).

31. Ekstrom M, Ahmadi Z, Bornefalk-Hermansson A, Abernethy A, Currow D. Oxygen for breathlessness in patients with chronic obstructive pulmonary disease who do not qualify for home oxygen therapy. Cochrane Database of Systematic Reviews. 2016;11:CD006429.

32. Booth S, Kelly MJ, Cox NP, Adams L, Guz A. Does oxygen help dyspnea in patients with cancer? American Journal of Respiratory and Critical Care Medicine. 1996;153(5):1515-8.

33. Higgins JPT, Green S. Cochrane Handbook for Systematic Reviews of Interventions Version 5.1.0 [updated March 2011]. 2011. In: The Cochrane Collaboration [Internet]. Available from www.handbook.cochrane.org. .

34. Moher D, Liberati A, Tetzlaff J, Altman DG. Preferred reporting items for systematic reviews and meta-analyses: the PRISMA statement2009 2009-07-21 10:46:49.

35. Bausewein C, Booth S, Gysels M, Higginson Irene J. Non-pharmacological interventions for breathlessness in advanced stages of malignant and non-malignant diseases. Cochrane Database of Systematic Reviews [Internet]. 2008; (2). 
36. Royal College of Physicians. Domiciliary oxygen therapy services: clinical guidelines and advice for prescribers. A report of the Royal College of Physicians. London: Royal College of Physicians; 1999.

37. Uronis H, McCrory Douglas C, Samsa G, Currow D, Abernethy A. Symptomatic oxygen for non-hypoxaemic chronic obstructive pulmonary disease. Cochrane Database of Systematic Reviews [Internet]. 2011; (6).

38. Report of the medical research council working party. Long term domiciliary oxygen in chronic hypoxic cor pulmonale complicating chronic bronchitis and emphysema. Lancet. 1981;1:6816.

39. Reeves BC, Deeks JJ, Higgins JPT, Wells GA. Chapter 13: Including non-randomized studies 2008.

40. Powers J, Bennett S. Measurement of dyspnea in patients treated with mechanical ventilation. American Journal of Critical Care. 1999;8(4):254-61.

41. Hjermstad MJ, Fayers PM, Haugen DF, Caraceni A, Hanks GW, Loge JH, et al. Studies comparing Numerical Rating Scales, Verbal Rating Scales, and Visual Analogue Scales for assessment of pain intensity in adults: a systematic literature review. J Pain Symptom Manage. 2011;41(6):107393.

42. Hozo S, Djulbegovic B, Hozo I. Estimating the mean and variance from the median, range, and the size of a sample. BMC Medical Research Methodology. 2005;5(1):13.

43. Deeks JJ, Higgins JPT, Altman DG. Chapter 9: Analysing data and undertaking meta-analyses The Cochrane Collaboration, ; 2011. .

44. Wong SL, Leong SM, Chan CM, Kan SP, Cheng HW. The Effect of Using an Electric Fan on Dyspnea in Chinese Patients With Terminal Cancer. American Journal of Hospice \& Palliative Medicine.34(1):42-6.

45. Eaton T, Fergusson W, Kolbe J, Lewis CA, West T. Short-burst oxygen therapy for COPD patients: a 6-month randomised, controlled study. European Respiratory Journal. 2006;27(4):697704.

46. Eves ND, Sandmeyer LC, Wong EY, Jones LW, Macdonald GF, Ford GT, et al. HeliumHyperoxia: A Novel Intervention To Improve the Benefits of Pulmonary Rehabilitation for Patients With COPD. Chest. 2009;135(3):609-18.

47. Jolly EC, Di Boscio V, Aguirre L, Luna CM, Berensztein S, Gené RJ. Effects of Supplemental Oxygen During Activity in Patients With Advanced COPD Without Severe Resting Hypoxemia. Chest. 2001;120(2):437-43.

48. Marciniuk DD, Butcher SJ, Reid JK, MacDonald GF, Eves ND, Clemens R, et al. The Effects of Helium-Hyperoxia on 6-mm Walking Distance in COPD: A Randomized, Controlled Trial. CHEST. 2007;131(6):1659-65.

49. McDonald CF, Blyth CM, Lazarus MD, Marschner I, Barter CE. Exertional oxygen of limited benefit in patients with chronic obstructive pulmonary disease and mild hypoxemia. American Journal of Respiratory and Critical Care Medicine [Internet]. 1995; (5 Pt 1):[1616-9 pp.].

50. Moore RP, Berlowitz DJ, Denehy L, Pretto JJ, Brazzale DJ, Sharpe K, et al. A randomised trial of domiciliary, ambulatory oxygen in patients with COPD and dyspnoea but without resting hypoxaemia. Thorax [Internet]. 2011; (1):[32-7 pp.].

51. Philip J, Gold M, Milner A, Di lulio J, Miller B, Spruyt O. A Randomized, Double-Blind, Crossover Trial of the Effect of Oxygen on Dyspnea in Patients with Advanced Cancer. Journal of Pain and Symptom Management. 2006;32(6):541-50.

52. Scorsone D, Bartolini S, Saporiti R, Braido F, Baroffio M, Pellegrino R, et al. Does a LowDensity Gas Mixture or Oxygen Supplementation Improve Exercise Training in COPD? Chest. 2010;138(5):1133-9.

53. Wadell K, Henriksson-Larsen K, Lundgren R. Physical training with and without oxygen in patients with chronic obstructive pulmonary disease and exercise-induced hypoxaemia. Journal of rehabilitation medicine. 2001;33(5):200-5. 
54. Johnson MJ, Bland JM, Oxberry SG, Abernethy AP, Currow DC. Clinically Important Differences in the Intensity of Chronic Refractory Breathlessness. Journal of Pain and Symptom Management. 2013;46(6):957-63.

55. Ries A. Minimally clinically important difference for the UCSD Shortness of Breath Questionnaire, Borg Scale, and Visual Analog Scale. Journal of Chronic Obstructive Pulmonary Disease. 2005;2:105-10.

56. Johnson MJ YJ, Hansen-Flaschen J, Lansing R, Ekstrom M, Similowski T, Currow D. . Towards an expert consensus to delineate a clinical syndrome of chronic breathlessness. . European Respiratory Journal 2017.

57. Luckett T, Phillips J, Johnson MJ, Farquhar M, Swan F, Assen T, et al. Contributions of a handheld fan to self-management of chronic breathlessness. European Respiratory Journal. 2017;50(2).

58. Currow DC, Abernethy AP, Johnson MJ. Activity as a Measure of Symptom Control. Journal of Pain and Symptom Management. 2012;44(5):e1-e2.

59. Maddocks M, Taylor V, Klezlova R, England R, Manderson C, Wilcock A. When will I get my breath back? Recovery time of exercise-induced breathlessness in patients with thoracic cancer. Lung cancer. 2012;76(1):128-9.

60. Johnson MJ, Simpson M, Millman R, Green G. Magnetoencephalography as a neuro-imaging method in chronic dyspnoea: A feasibility study. European Respiratory Journal. 2014;44(Suppl. 58):P670.

61. Baltzan MA, Alter A, Rotaple M, Kamel H, Wolkove N. Fan to palliative exercise-induced dyspnoea with severe COPD. Journal of respiratory and critical care medicine. 2000;161(3 supplement):A59.

62. Marchetti N, Travaline J, Criner G. Air current applied to the face of COPD patients enhances leg ergometry performance. Am J Resp and Crit Care. 2004;169:A773.

63. Resnick B SA. Understanding what motivates older adults to exercise Journal of gerontological nursing. 2000;26(3):34-41.

64. Keating A, Lee A, Holland AE. What prevents people with chronic obstructive pulmonary disease from attending pulmonary rehabilitation? A systematic review. Chronic Respiratory Disease. 2011;8(2):89-99.

65. Fischer MJ, Scharloo M, Abbink JJ, van 't Hul AJ, van Ranst D, Rudolphus A, et al. Drop-out and attendance in pulmonary rehabilitation: The role of clinical and psychosocial variables. Respiratory Medicine. 2009;103(10):1564-71.

66. Ries AL, Kaplan RM, Myers R, Prewitt LM. Maintenance after pulmonary rehabilitation in chronic lung disease: a randomized trial. Am J Respir Crit Care Med. 2003;167(6):880-8.

67. Heppner PS, Morgan C, Kaplan RM, Ries AL. Regular walking and long-term maintenance of outcomes after pulmonary rehabilitation. Journal of Cardiopulmonary Rehabilitation. 2006;26(1):4453.

68. Marchetti N, Lammi MR, Travaline JM, Ciccolella D, Civic B, Criner GJ. Air Current Applied to the Face Improves Exercise Performance in Patients with COPD. Lung. 2015;193(5):725-31.

69. Farquhar MC, Prevost AT, McCrone P, Brafman-Price B, Bentley A, Higginson IJ, et al. Is a specialist breathlessness service more effective and cost-effective for patients with advanced cancer and their carers than standard care? Findings of a mixed-method randomised controlled trial. BMC Medicine. 2014;12(1):194.

70. Higgins JP, Thompson SG. Quantifying heterogeneity in a meta-analysis. Statistics in Medicine. 2002;21(11):1539-58. 